\title{
Comment modéliser les écoulements diphasiques compressibles sur des grilles hybrides?
}

\author{
L. Jeannin ${ }^{1}$, I. Faille' et T. Gallouët ${ }^{2}$ \\ 1 Institut français du pétrole, 1 et 4, avenue de Bois-Préau, 92852 Rueil-Malmaison Cedex - France \\ 2 CMI, Université de Provence, 39, rue Joliot-Curie, 13453 Marseille Cedex - France \\ e-mail : laurent.jeannin@ifp.fr - isabelle.faille@ifp.fr - thierry.gallovet@cmi.univ-mrs.fr
}

Résumé - Les maillages hybrides offrent la flexibilité nécessaire pour modéliser les écoulements en milieu poreux à travers des structures géologiques de géométrie complexe. L'objet de cette étude est de développer des approches numériques performantes pour simuler les écoulements diphasiques compressibles en milieu poreux sur ces maillages hybrides.

Mots-clés : réservoir, volumes finis, éléments finis mixtes, écoulement diphasique.

\begin{abstract}
How to Model Compressible Two-Phase Flows on Hybrid Grids? - Hybrid grids allow to model flows in porous media through geometrically complex geological features. The purpose of this paper is to develop efficient numerical methods to simulate compressible two-phase flows on these hybrid grids.

Keywords: reservoir, finite volume methods, mixed finite element methods, two-phase flow.
\end{abstract}




\section{INTRODUCTION}

Une description précise des écoulements dans les réservoirs souterrains d'hydrocarbures lors de leur exploitation nécessite de prendre en compte la complexité géométrique des structures géologiques. Les maillages hybrides (c'est-à-dire les maillages dont les mailles sont de natures géométriques différentes) permettent un pavage de l'espace d'un réservoir géométriquement complexe. On développe et compare dans cette étude deux approches numériques différentes pour discrétiser le système d'équations décrivant les écoulements diphasiques compressibles sur des maillages hybrides à deux et trois dimensions.

Nous rappelons les deux approches numériques principales concernant la discrétisation spatiale dans le cas monophasique faiblement compressible. Pour la modélisation du problème diphasique, la première approche étudiée est une approche par volumes finis; nous avons généralisé à trois dimensions sur des maillages flexibles les schémas à continuité de flux. La seconde approche consiste à utiliser les fonctions de base de l'espace de Raviart-Thomas de plus bas degré pour évaluer les termes diffusifs du système d'équations aux dérivés partielles.

\section{LE CAS MONOPHASIQUE}

\subsection{Le problème physique}

Soit $\Omega$ un domaine borné de $\boldsymbol{R}^{d}, \Gamma$ sa frontière, $\Gamma_{D}$ la partie de $\Gamma$ sur laquelle sont appliquées des conditions de Dirichlet, $\Gamma_{N}$ la partie de $\Gamma$ sur laquelle sont appliquées des conditions de Neumann $\left(\Gamma_{D} \cap \Gamma_{N}=\varnothing, \Gamma_{D} \cup \Gamma_{N}=\Gamma\right)$.

Le problème physique décrivant l'écoulement d'un fluide faiblement compressible de compressibilité $c(c \Delta P<<1, \Delta P$ caractérise l'ordre de grandeur de la chute de pression dans le réservoir), de viscosité $\mu$ dans un milieu poreux caractérisé par une porosité $\Phi$ et une perméabilité $\boldsymbol{K}$, constitue un problème parabolique en pression qui se met sous la forme :

$$
\begin{gathered}
\Phi_{c} \frac{\partial P}{\partial t}+\nabla \cdot \boldsymbol{u}=f \\
\boldsymbol{u}=-\frac{\boldsymbol{K}}{\mu} \nabla P \\
\boldsymbol{u} \cdot \boldsymbol{n}=g \operatorname{sur} \Gamma_{N} \\
P=P_{D} \operatorname{sur} \Gamma_{D}
\end{gathered}
$$

La première de ces équations exprime la conservation de la masse, la seconde constitue la loi de Darcy.

Pour la discrétisation spatiale du problème, on note $\Sigma_{h}$ la partition de $\Omega$ en simplexes $K$ ( $h$ est le diamètre maximal des mailles), $\sigma_{h}$ l'ensemble des arêtes de $\Sigma_{h}$.

\subsection{L'approximation volumes finis (VF)}

La loi de conservation de la masse est intégrée maille par maille ; l'inconnue à déterminer (ici la pression) est supposée constante par maille. On se ramène alors à la détermination des flux sur les bords des mailles, c'est-à-dire à l'évaluation de $-\int_{\partial K} \boldsymbol{K} \nabla P \cdot \boldsymbol{n} \mathrm{d} l$ sur l'ensemble des arêtes de $\Sigma_{h}$.

\subsubsection{L'approximation du flux}

L'approche par volumes finis centrés sur les mailles vérifie des propriétés de consistance. L'approximation :

$$
\frac{F_{\delta}}{l_{\delta}} \text { de }-\frac{1}{l_{\delta}} \int \boldsymbol{K} \nabla P \cdot \boldsymbol{n}_{\delta} \mathrm{d} l
$$

sur l'arête $\delta$ de longueur $l_{\delta}$ est dite consistante si :

$$
\frac{F_{\delta}}{l_{\delta}}=-\frac{1}{l_{\delta}} \int_{\delta} \boldsymbol{K} \nabla P \cdot \boldsymbol{n}_{\delta} \mathrm{d} l+o(1) .
$$

Le schéma est conservatif : on utilise une même approximation du flux de part et d'autre de l'arête.

L'approximation $F_{\delta}$ du flux sur l'arête $\delta$ se met sous la forme $F_{\delta}=\Sigma_{j} \alpha_{j} P_{j}$ (j désignant des mailles voisines de l'arête $\delta$ ). La discrétisation des équations de conservation sur chaque maille amène à la résolution d'un système linéaire $\mathcal{H} \mathscr{P}=\mathcal{D}$ de dimension $\operatorname{card}\left(\Sigma_{h}\right)$, le nombre de mailles du maillage.

Pour un maillage quelconque, on a le résultat suivant (Faille, 1992) : un schéma volumes finis, centrés sur les mailles, conservatif avec une approximation consistante des flux, est tel que son erreur de consistance tend faiblement vers 0 avec le pas du maillage (l'erreur de consistance est l'erreur avec laquelle la solution continue vérifie l'équation discrétisée).

Cette approche est une généralisation de l'approximation à deux points sur les maillages dits orthogonaux qui vérifient les deux propositions suivantes :

- l'ensemble des centres des mailles $K$ du maillage est tel que, pour chaque maille $x_{k}$, la droite joignant les centres de maille $x_{K}$ et $x_{L}$ est orthogonale à l'arête $\sigma=K \cap L$;

- pour les arêtes de bord, la droite passant par le centre de la maille de bord et orthogonale à l'arête de bord intersecte cette arête de bord. Sous ces conditions et en supposant la perméabilité homogène et isotrope, l'approximation volumes finis de l'équation parabolique converge (i.e. dans $\left.L^{2}(\Omega)\right)$ (Eymard et al., 1996).

\subsubsection{Schéma en $O$}

Le schéma à continuité de flux de type $\mathrm{O}$ est un schéma de type volumes finis adapté aux grilles hybrides, qui construit une approximation consistante sur des maillages hybrides pour des milieux caractérisés par des tenseurs de perméabilité complets. La construction de ces approximations 2D a été proposée par Aavatsmark (1996, 1998a, 1998b). Nous avons 
généralisé cette approche pour des maillages hybrides 3D, composés de mailles polyédriques. Le flux $F_{k}=\Sigma_{j}\left(\alpha_{j} P_{j}\right)$ à travers une face est approché linéairement à partir des pressions au centre des mailles ayant un sommet commun avec la face. Le calcul des coefficients $\alpha_{j}$ est explicité en annexe.

La convergence d'une telle approximation est avant tout étudiée numériquement. Pour un tenseur de perméabilité homogène, le schéma peut donner une approximation à deux points si le maillage est orthogonal. C'est uniquement dans ce cadre que l'on montre l'existence et l'unicité de la solution discrète, $\mathcal{H}$ étant une $M$ matrice (la définition d'une $M$ matrice est rappelée en annexe).

\subsection{L'approximation éléments finis mixtes (EFM)}

La formulation mixte résout un problème variationnel équivalent à 1.1. Elle introduit les espaces fonctionnels de Hilbert suivants :

$$
\begin{gathered}
W=L^{2}(W) \\
V=H(\Omega, \operatorname{div})=\left\{\boldsymbol{u}, \boldsymbol{u} \in\left(L^{2}(\Omega)\right)^{d}, \nabla \cdot \boldsymbol{u} \in L^{2}(\Omega)\right\}
\end{gathered}
$$

avec :

$$
\|\boldsymbol{u}\|=\left(\|\boldsymbol{u}\|^{2}+\|\nabla \cdot \boldsymbol{u}\|^{2}\right)^{1 / 2}
$$

On définit également :

$$
\begin{gathered}
H_{0, N}(\Omega, \operatorname{div})=\left\{\boldsymbol{v}, \boldsymbol{v} \in H(\Omega, \operatorname{div}), \boldsymbol{v} \cdot \boldsymbol{n}=0 \quad \text { sur } \quad \Gamma_{N}\right\} \\
H_{g, N}(\Omega, \operatorname{div})=\left\{\boldsymbol{u}, \boldsymbol{u} \in H(\Omega, \operatorname{div}), \boldsymbol{u} \cdot \boldsymbol{n}+g=0 \text { sur } \quad \Gamma_{N}\right\}
\end{gathered}
$$

Résoudre le problème 1.1 est équivalent à trouver $(p, \boldsymbol{u}) \in W \times H_{g, N}(\Omega$, div), vérifiant :

$$
\begin{gathered}
\int_{\Omega} \mu \boldsymbol{u} \boldsymbol{K}^{-1} \boldsymbol{u} \mathrm{d} x-\int_{\omega} p \nabla \cdot \boldsymbol{v} \mathrm{d} x=-\int_{\Gamma_{D}} p_{d} \boldsymbol{v} \cdot \boldsymbol{n} \mathrm{d} \boldsymbol{\sigma}, \forall \boldsymbol{v} \in H_{0, N}(\Omega, \mathrm{div}) \\
\int_{\omega} \Phi_{c} \frac{\partial p}{\partial t} q \mathrm{~d} x+\int_{\Omega} q \nabla \cdot \boldsymbol{u}=\int_{\Omega} f q \mathrm{~d} x \quad \forall q \in L^{2}(\Omega)
\end{gathered}
$$

$\operatorname{avec} p(x, 0)=p^{0}, p^{0} \in L^{2}(\Omega)$.

Pour la résolution discrète du problème variationnel, on construit les espaces de dimension finie $V_{h}$ et $W_{h}$, denses respectivement dans $V$ et $W$. On utilise dans cette étude les espaces de Raviart-Thomas de degré le plus bas, et un schéma d'Euler implicite pour la discrétisation en temps.

Une approximation utilisant l'espace des vitesses $H(\Omega$, div) est donnée par l'espace de Raviart-Thomas $R T_{0}(\Omega) \cdot R T_{0}(\Omega)=\left\{v \in H(\Omega, \operatorname{div}), v_{\left.\right|_{K}} \in R T_{0}(K), K \in \Sigma_{h}\right\}$, où pour toute maille $K$, on définit :

$$
R T_{0}(K)=\left\{P_{0}(K)^{2} \oplus x \cdot P_{0}(K) x \in \boldsymbol{R}^{d}\right\}
$$

$\left(P_{0}(K)\right.$ désigne l'ensemble des polynômes constants définis $\operatorname{sur} K)$.
Un choix approprié des degrés de liberté pour $R T_{0}$ assure la continuité de la composante normale de la vitesse sur le bord des éléments. L'espace approchant la pression est l'espace des fonctions constantes par maille.

La discrétisation du problème conduit à la résolution d'un problème de point selle en $\left(\boldsymbol{u}_{h}, p_{h}\right)$, qui s'écrit sous forme matricielle:

$$
\left(\begin{array}{cc}
\mathcal{A} & \mathcal{B} \\
-{ }^{t} \mathcal{B} & \mathcal{C}
\end{array}\right)\left(\begin{array}{l}
\mathcal{U} \\
\mathcal{P}
\end{array}\right)=\left(\begin{array}{l}
\mathcal{F} \\
\mathcal{G}
\end{array}\right)
$$

$\mathcal{A}$ est la matrice de raideur des vitesses.

L'approche EFM converge lorsque le pas du maillage tend vers 0 (Brezzi et Fortin, 1991), et il n'y a pas de contraintes sur les déformations du maillage.

Notons que sous certaines conditions restrictives concernant le maillage, une variante de la méthode des éléments finis mixtes, dite méthode mixte étendue, permet de construire une classe de schémas volumes finis pour des tenseurs de perméabilité complets dont on déduit la convergence de l'approche par éléments finis mixtes (sous certaines conditions concernant le maillage) (Aarbogast et al., 1997; Wheeler, 1997).

\subsection{Cas tests}

Les deux premiers tests permettent d'étudier le bon comportement des schémas volumes finis dits à continuité de flux en $O$ sur des maillages hybrides en régime permanent et en monophasique, et de comparer la solution obtenue à celle fournie par les EFM.

\subsubsection{Consistance du schéma VF}

On étudie le comportement de la solution en fonction de la déformation du maillage. Le fluide est supposé incompressible $(c=0)$, et la perméabilité homogène et constante sur tout le domaine. On considère alors le problème suivant :

$$
\left\{\begin{array}{l}
-\Delta P=0 \text { dans } \Omega=] 0 ; 10\left[^{3}\right. \\
P(0, y, z)=0 \\
P(10, y, z)=10 \\
\nabla P(x, 0, z) \cdot \boldsymbol{n}_{\partial \Omega}=\nabla P(x, 10, z) \cdot \boldsymbol{n}_{\partial \Omega}=0 \\
\nabla P(x, y, 0) \cdot \boldsymbol{n}_{\partial \Omega}=\nabla P(x, y, 10) \cdot \boldsymbol{n}_{\partial \Omega}=0
\end{array}\right.
$$

dont la solution exacte est : $\overline{\boldsymbol{u}}(x, y, z)=x$.

L'espace est maillé à l'aide de tétraèdres par une tétraédrisation de Delaunay (fig. 1). L'erreur entre la solution approchée et la solution exacte est de l'ordre de la précision de la machine pour le maillage ci-dessus.

On étudie ensuite l'effet de grandes déformations du maillage. Pratiquement, on divise les coordonnées suivant l'axe des $z$ par un facteur arbitraire $\alpha$. Le calcul des flux par le schéma est $\mathrm{O}$ consistant, mais contrairement à un schéma 


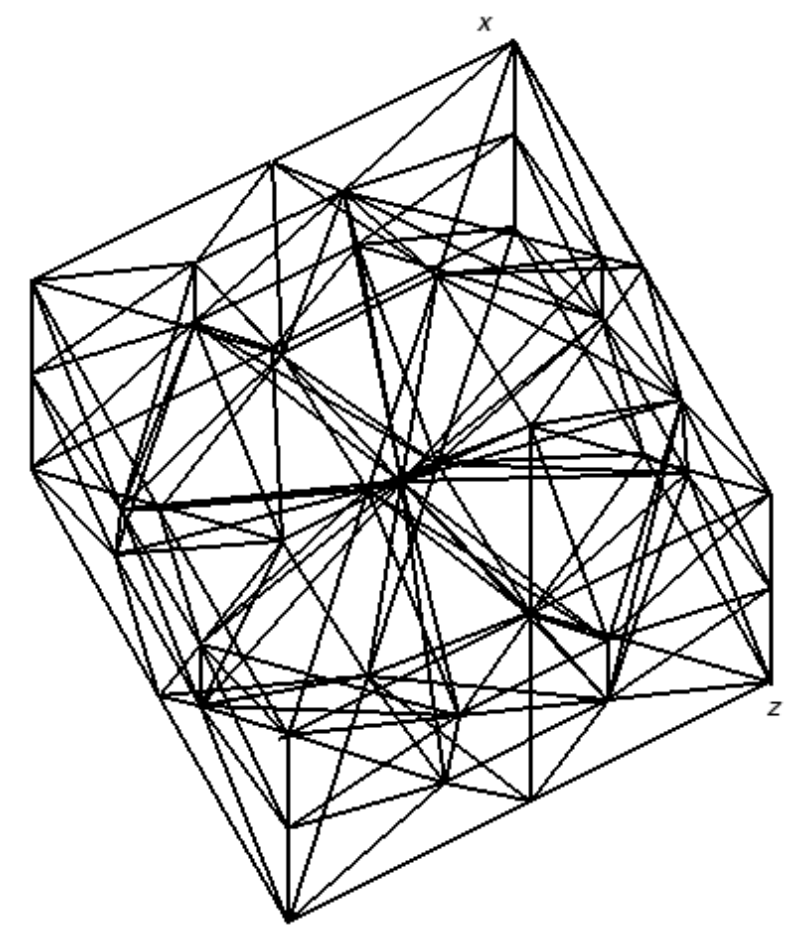

Figure 1

Maillage tétraédrique 3D.

Tetrahedral $3 D$ grid.

VF à deux points sur un maillage orthogonal, on ne possède aucune propriété de stabilité et de convergence dans le cas général.

Pratiquement, les tests numériques montrent que la structure de la matrice pose des problèmes de convergence au niveau du solveur pour les très grandes déformations; on perd la convergence pour $\alpha$ de l'ordre de 100 ; on s'écarte alors très fortement des tétraédrisations de Delaunay délivrées par les mailleurs en pratique. À $2 \mathrm{D}$, le schéma à continuité de flux en $\mathrm{O}$ permet de traiter des maillages de type Delaunay (tenseur homogène isotrope) et des maillages triangulaires réguliers avec un rapport d'anisotropie de l'ordre de 1/10 (Aavastmark, 1998b).

À $3 \mathrm{D}$, nous avons vérifié la convergence numérique du schéma en $\mathrm{O}$ sur différents maillages tétraédriques (la tétraédrisation 3D est une tétraédrisation de type Delaunay, elle ne satisfait en général aucun critère d'optimisation en ce qui concerne les angles des tétraèdres) et sur des maillages composés de prismes et d'hexaèdres. On peut également se reporter aux tests de Aavatsmark et al. (1997) pour des cas pratiques sur grilles hexaédriques.

\subsubsection{Convergence numérique du schéma VF comparé à un schéma EFM en milieu hétérogène}

Le deuxième problème résolu est une étude de convergence 2D sur un maillage triangulaire en milieu hétérogène.
Le tenseur de perméabilité est anisotrope et donné par :

$$
\begin{aligned}
& \boldsymbol{K}=K_{0}\left(\begin{array}{cc}
y(y-10)+1 & 0 \\
0 & x(x-10)+1
\end{array}\right) \\
& \left\{\begin{array}{l}
-\nabla \cdot(K \nabla u)=f \text { dans } \Omega=] 0 ; 10\left[^{3}\right. \\
f=2(y(y+1)(y-10)+x(x+1)(x-10)) \\
u=0 \text { sur } \partial \Omega
\end{array}\right.
\end{aligned}
$$

La solution $\bar{u}$ du problème est : $\bar{u}=x(x-10) y(y-10)$.

Nous précisons l'évolution (tableau 1) avec le pas du maillage du carré de la norme $L^{2}$ de l'erreur : $\Sigma_{k} V_{k}\left(u_{k}-\bar{u}_{k}\right)^{2}$ et l'erreur absolue maximale : $\sup _{k}\left(u_{k}-\bar{u}_{k}\right)$.

\section{TABLEAU 1}

Comparaison VF/EFM

Comparison FVIMFE

\begin{tabular}{l|c|c|c|c}
\hline & err abs VF & err abs EFM & err $L^{2}$ VF & err $L^{2}$ EFM \\
\hline$h$ & 26,25 & 27,63 & 139,47 & 84,46 \\
\hline$h / 2$ & 7,49 & 5,02 & 30,78 & 21,32 \\
\hline$h / 4$ & 2,66 & 1,70 & 9,34 & 6,37 \\
\hline
\end{tabular}

Comme précédemment, les résultats obtenus avec la méthode des éléments finis mixtes sont meilleurs que les résultats obtenus avec celle des volumes finis. Cependant, on met en évidence sur ces exemples la convergence des schémas volumes finis à continuité de flux lorsque le pas du maillage diminue. Une telle étude incite à généraliser ces deux approches dans le cas d'écoulements polyphasiques compressibles.

\section{LE MODĖLE DIPHASIQUE}

Les équations de conservation de la phase huile et de la phase eau s'écrivent :

$$
\begin{gathered}
\frac{\partial}{\partial t}\left(\phi \rho_{w} S_{w}\right)+\nabla \cdot\left(\rho_{w} \frac{k_{r, w}}{\mu_{w}} \boldsymbol{K} \nabla P_{w}\right)=f_{w} \\
\frac{\partial}{\partial t}\left(\phi \rho_{o} S_{o}\right)+\nabla \cdot\left(\rho_{o} \frac{k_{r, o}}{\mu_{o}} \boldsymbol{K} \nabla P_{o}\right)=f_{o} \\
P_{o}-P_{w}=P_{c}\left(S_{w}\right) \\
S_{o}+S_{w}=1
\end{gathered}
$$

avec :

$\Phi$ porosité du milieu poreux

$\boldsymbol{K}$ tenseur de perméabilité du milieu poreux

$\rho_{i} \quad$ densité de la phase $i$

$\mu_{i} \quad$ viscosité de la phase $i$

$P_{i} \quad$ pression de la phase $i$ 
$k_{r, i}$ perméabilité relative de la phase $i$, fonction de la saturation dans la phase $i$

$S_{i} \quad$ saturation de la phase $i$

$f_{i} \quad$ terme source de la phase $i$

$\lambda_{i}=\frac{k_{r, i}}{\mu_{i}}$ définit la mobilité dans la phase $i$

$P_{c} \quad$ pression capillaire.

Le système d'équations décrivant les écoulements diphasiques compressibles constitue un système d'équations différentielles aux dérivées partielles couplées.

Les temps caractéristiques de diffusion liés à la compressibilité des fluides : $\tau_{p}=\frac{c \mu L^{2}}{K}$ et à la diffusion capillaire : $\tau_{s}=\frac{\mu L^{2}}{\boldsymbol{K} P_{c}}$ sont généralement très différents, ce qui peut justifier l'emploi de l'approximation incompressible. $L$ est une longueur caractéristique de la simulation, la taille des mailles par exemple; $c$ est caractéristique de l'ordre de grandeur de la compressibilité des fluides : $c_{i}=\left(\frac{\partial \ln \rho_{i}}{\partial P_{i}}\right) V_{i, T}$.

Cependant, dans les applications à des réservoirs, on souhaite avoir une description qui traite à la fois les temps courts où les phénomènes de compressibilité des fluides dominent (récupération primaire et tests de puits), et les phénomènes liés à la diffusion de la pression capillaire (cas de la récupération secondaire), ce qui nécessite le développement d'approches numériques en compressible.

On met en œuvre, dans cette étude, un schéma d'Euler implicite en temps et deux approches différentes pour la discrétisation spatiale.

\subsection{L'approximation volumes finis}

Les lois de conservation de chacune des phases sont intégrées maille par maille; les inconnues à déterminer (ici $P_{w}$ et $S_{w}$ ) sont supposées constantes par maille. On se ramène alors à la détermination des flux sur les bords des mailles, c'est-à-dire à l'évaluation de $Q_{w}=\int_{\partial K}\left(\rho_{w} \lambda_{w} K \nabla P_{w}\right) \cdot n_{\partial K}$ et $Q_{o}$, les flux massiques de chacune des phases.

Les mobilités $\lambda_{i}$ sur l'arête $\delta$ (la face 3D) sont évaluées par un schéma amont couramment utilisé dans le milieu pétrolier (discrétisation consistante d'une équation hyperbolique à l'ordre le plus bas). $\rho_{i}$, densité de la phase $i$, est évaluée sur l'arête des mailles à 2D (la face 3D).

L'approximation de $\frac{1}{l_{\delta}} \int \boldsymbol{K} \nabla P \cdot \boldsymbol{n}_{\delta} \mathrm{d} l$ sur l'arête $\delta$ est construite de manière consistante $(c f .1 .2 .1)$ pour chacune des phases.

La taille du système à résoudre est $2 \operatorname{card}\left(\Sigma_{h}\right)$. D'autre part, une telle discrétisation se généralise facilement à des descriptions physiques plus complexes (modèle black oil, modèle compositionnel, etc.) (Lemonnier, 1991).

\subsection{L'approximation éléments finis mixtes}

\subsubsection{Le cas de fluides incompressibles}

Dans le cas de fluides incompressibles, on introduit comme variables principales la variable de pression globale $p_{t}$ et la saturation de l'une des phases (Chavent et Jaffré, 1987) :

$$
p_{t}=p_{w}+\int_{S_{c}}^{S} \frac{\lambda_{o}}{\lambda_{t}} \frac{\mathrm{d} P_{c}}{\mathrm{~d} S} \mathrm{~d} s
$$

$\lambda_{t}=\lambda_{o}+\lambda_{w}$ définit la mobilité totale. L'avantage de ce changement de variables est qu'il permet de se ramener à une équation elliptique en pression globale et à une équation en saturation de type parabolique dégénérée dans le cas de fluides incompressibles, que l'on peut résoudre par une méthode de splitting. Les deux équations sont alors découplées (de plus, le flux volumique total $u_{t}$ est une grandeur continue - Pfertzel, 1987 - contrairement au flux massique dans le cas de fluides compressibles) :

$$
\begin{gathered}
\nabla \cdot u_{t}=0 \\
\phi \frac{\partial}{\partial t} S_{w}+\nabla \cdot\left(\frac{\lambda_{w}}{\lambda_{t}} u_{t}+\frac{\lambda_{o} \lambda_{w}}{\lambda_{t}} u_{s}\right)=f_{w} \\
u_{t}=-\lambda_{t} K \nabla P_{t} \\
u_{s}=-K \nabla P_{c}(S)
\end{gathered}
$$

Cette formulation en pression globale a été introduite par Chavent et Jaffré (1987). Ils résolvent l'équation elliptique en pression globale par une méthode d'éléments finis mixtes hybrides, et utilisent une méthode où la saturation est obtenue à chaque pas de temps en deux étapes : la première étape est un calcul par éléments finis (éléments finis discontinus pour les termes d'advection, éléments finis mixtes pour les termes de diffusion liés à la capillarité), la seconde étape consiste en l'utilisation d'un limiteur de pentes.

\subsubsection{Le cas de fluides compressibles}

Dans le cas de fluides compressibles, les deux équations en pression globale et en saturation restent couplées.

Une possibilité est de se placer dans l'approximation des fluides faiblement compressibles afin de découpler les deux équations et de supposer que la dérivée temporelle du terme de pression capillaire est négligeable devant la dérivée temporelle d'une pression moyenne $p_{g}$ caractérisant l'état thermodynamique des fluides. Cette approximation est justifiée en particulier dans le cas $c_{i} \Delta P_{i}<<1$ ( $i$ désignant chacune des phases) et donc dans des conditions réservoirs standard. Néanmoins, pour des problèmes diphasiques avec des 
fluides plus compressibles (huile-gaz) par exemple, cette approximation est injustifiée.

Durlofsky (1993) introduit dans le cadre des approximations précédentes la variable de pression moyenne $p_{g}$ définie comme suit : $p_{g}=1 / 2\left(P_{w}+P_{0}\right)$; l'équation d'état de chacun des fluides est alors fonction de cette pression moyenne. Ces hypothèses simplificatrices lui permettent de se ramener à une équation parabolique en pression globale et à une équation parabolique dégénérée en saturation. Ce système d'équations est résolu de manière séquentielle par un schéma EFM en pression et une approximation volumes finis explicite en temps pour la saturation.

Une autre approche dans le cas de fluides compressibles (Bergamashi et al., 1998) consiste à découpler les équations en supposant que l'équation de conservation $S_{w}+S_{o}=1$ n'est pas exactement satisfaite. Cette formulation introduit une erreur en $O(\Delta t)$ dans la conservation du volume global. On se ramène à la résolution implicite d'une équation en pression et de deux équations hyperboliques pour chacune des équations de conservation, résolues avec des méthodes de type Godunov d'ordre élevé.

\subsubsection{La méthode proposée}

Le fait qu'il ne soit pas possible, dans le cas général, de découpler les équations nous a amenés à développer une approche proposée par Gallouët (1999), conservative et n'impliquant aucune hypothèse physique restrictive.

Une première formulation consiste à utiliser les fonctions de base des éléments finis mixtes pour évaluer les termes $\boldsymbol{v}_{w}=-\boldsymbol{K} \nabla P_{w}$ et $\boldsymbol{v}_{o}=-\boldsymbol{K} \nabla P_{o}$, les mobilités étant évaluées par un schéma amont, les densités sur les arêtes.

Une telle méthode permet $a$ priori de traiter des maillages destructurés de manière plus performante que les schémas volumes finis : les EFM apportent la flexibilité en ce qui concerne le choix du maillage.

On note $v_{\sigma}$ la fonction de base relative à l'arête $\sigma \mathrm{du}$ maillage $\Sigma_{h}$.

$$
\begin{array}{r}
\boldsymbol{v}_{o} \text { et } \boldsymbol{v}_{w} \text { s'écrivent } \boldsymbol{v}_{i}=\Sigma_{\sigma \in \sigma_{h}} u_{\sigma, i} v_{\sigma} \text { avec : } \\
\Sigma_{\bar{\sigma}} u_{\sigma, w} \int \boldsymbol{v}_{\sigma} \boldsymbol{K}^{-1} \boldsymbol{v}_{\bar{\sigma}} \mathrm{d} \Omega=l_{\sigma}\left(P_{L, w}-P_{K, w}\right) \\
\Sigma_{\bar{\sigma}} u_{\sigma, o} \int \boldsymbol{v}_{\sigma} \boldsymbol{K}^{-1} \boldsymbol{v}_{\bar{\sigma}} \mathrm{d} \Omega=l_{\sigma}\left(P_{L, o}-P_{K, o}\right)
\end{array}
$$

(L'arête $\sigma$ définie comme l'intersection des mailles $K$ et $L$ est orientée de $L$ vers $K$ ). La discrétisation adoptée pour les deux équations de conservation est la suivante:

$$
\begin{aligned}
\frac{S_{k, w}^{n+1}-S_{k, w}^{n}}{\Delta t}+ & \frac{1}{\Phi \Delta t \rho_{k, w}^{n+1}} S_{k, w}^{n}\left(\rho_{k, w}^{n+1}-\rho_{k, w}^{n}\right) \\
& +\Sigma_{i \in \partial K} \frac{\rho_{i, w}^{n+1}}{\Phi \rho_{k, w}^{n+1}}\left(\lambda_{i, w}^{n+1} u_{i, w}^{n+1}\right)=\frac{f_{k, w}^{n+1}}{\Phi \rho_{k, w}^{n+1}} \\
\frac{S_{k, o}^{n+1}-S_{k, o}^{n}}{\Delta t}+ & \frac{1}{\Phi \Delta t \rho_{k, o}^{n+1}} S_{k, o}^{n}\left(\rho_{k, o}^{n+1}-\rho_{k, o}^{n}\right) \\
& +\Sigma_{i \in \partial K} \frac{\rho_{i, o}^{n+1}}{\Phi \rho_{k, o}^{n+1}}\left(\lambda_{i, o}^{n+1} u_{i, o}^{n+1}\right)=\frac{f_{k, o}^{n+1}}{\Phi \rho_{k, o}^{n+1}}
\end{aligned}
$$

Les inconnues du système sont $P_{k, w}, S_{k, w}\left(\right.$ ou $\left.P_{k, o}, S_{k, o}\right)$, $u_{\sigma, o}, u_{\sigma, w}$. La taille du système non linéaire à résoudre est $2\left(\operatorname{card}\left(\Sigma_{h}\right)+\operatorname{card}\left(\sigma_{h}\right)\right)$.

L'approche que nous avons développée peut également être écrite avec les variables $p_{t}$ et $S_{w}$. Dans le cas général de deux fluides compressibles, on obtient deux équations couplées :

$$
\begin{gathered}
\frac{\partial}{\partial t}\left(\phi \rho_{w} S_{w}\right)+\nabla \cdot\left(\rho_{w}\left(\frac{\lambda_{w}}{\lambda_{t}} u_{t}+\frac{\lambda_{o} \lambda_{w}}{\lambda_{t}} u_{s}\right)\right)=f_{w} \\
\frac{\partial}{\partial t}\left(\phi \rho_{o} S_{o}\right)+\nabla \cdot\left(\rho_{o}\left(\frac{\lambda_{o}}{\lambda_{t}} u_{t}-\frac{\lambda_{o} \lambda_{w}}{\lambda_{t}} u_{s}\right)\right)=f_{o}
\end{gathered}
$$

$u_{s}$ et $u_{t}$ sont évalués dans l'espace $R T_{0}(\Omega)$. On note $v_{\sigma}$ la fonction de base relative à l'arête $\sigma$ du maillage :

$$
\begin{gathered}
\Sigma_{\bar{\sigma}} u_{\sigma, t} \frac{\int \boldsymbol{v}_{\sigma} \boldsymbol{K}^{-1}}{\lambda_{t}} v_{\bar{\sigma}} \mathrm{d} \Omega=l_{\sigma}\left(P_{L, t}-P_{K, t}\right) \\
\Sigma_{\bar{\sigma}} u_{\sigma, s} \int \boldsymbol{v}_{\sigma} \boldsymbol{K}^{-1} \boldsymbol{v}_{\bar{\sigma}} \mathrm{d} \Omega=-l_{\sigma}\left(P_{c L, s}-P_{c K, s}\right)
\end{gathered}
$$

La discrétisation des équations de conservation s'écrit dans le cas de fluides compressibles selon les équations (1) et (2) ci-dessous.

La pression dans chacune des phases s'exprime alors comme la somme de la pression globale et d'une fonction de la saturation définie par une intégrale, qui peut être tabulée.

Nous avons adopté la première formulation développée dans ce paragraphe pour réaliser des tests numériques. La preuve de la convergence de cette approche, dans le cas de fluides incompressibles, sans pression capillaire et avec des perméabilités relatives égales aux saturations, est développée dans Dong et al. (1999).

$$
\begin{aligned}
& \frac{S_{k, w}^{n+1}-S_{k, w}^{n}}{\Delta t}+\frac{1}{\Phi \Delta t \rho_{k, w}^{n+1}} S_{k, w}^{n}\left(\rho_{k, w}^{n+1}-\rho_{k, w}^{n}\right)+\Sigma_{i \in \partial K} \frac{\rho_{i, w}^{n+1}}{\Phi \rho_{k, w}^{n+1}}\left(\frac{\lambda_{i, w}^{n+1}}{\lambda_{i, t}^{n+1}} u_{i, t}^{n+1}+\frac{\lambda_{i, o}^{n+1} \lambda_{i, w}^{n+1}}{\lambda_{i, t}^{n+1}} u_{i, s}^{n+1}\right)=\frac{f_{k, w}^{n+1}}{\Phi \rho_{k, w}^{n+1}} \\
& \frac{S_{k, o}^{n+1}-S_{k, o}^{n}}{\Delta t}+\frac{1}{\Phi \Delta t \rho_{k, o}^{n+1}} S_{k, o}^{n}\left(\rho_{k, o}^{n+1}-\rho_{k, o}^{n}\right)+\Sigma_{i \in \partial K} \frac{\rho_{i, o}^{n+1}}{\Phi \rho_{k, o}^{n+1}}\left(\frac{\lambda_{i, o}^{n+1}}{\lambda_{i, t}^{n+1}} u_{i, t}^{n+1}-\frac{\lambda_{i, o}^{n+1} \lambda_{i, w}^{n+1}}{\lambda_{i, t}^{n+1}} u_{i, s}\right)=\frac{f_{k, o}^{n+1}}{\Phi \rho_{k, o}^{n+1}}
\end{aligned}
$$




\section{3 Étude d'un balayage en diphasique compressible}

\subsection{1 Étude d'un maillage 1D régulier}

On considère un maillage $1 \mathrm{D}$ composé de 10 mailles carrées de côté $1 \mathrm{~m}$ (ce maillage est orthogonal). On réalise un balayage d'un réservoir d'huile par de l'eau. Le profil des saturations est représenté en figure 2.
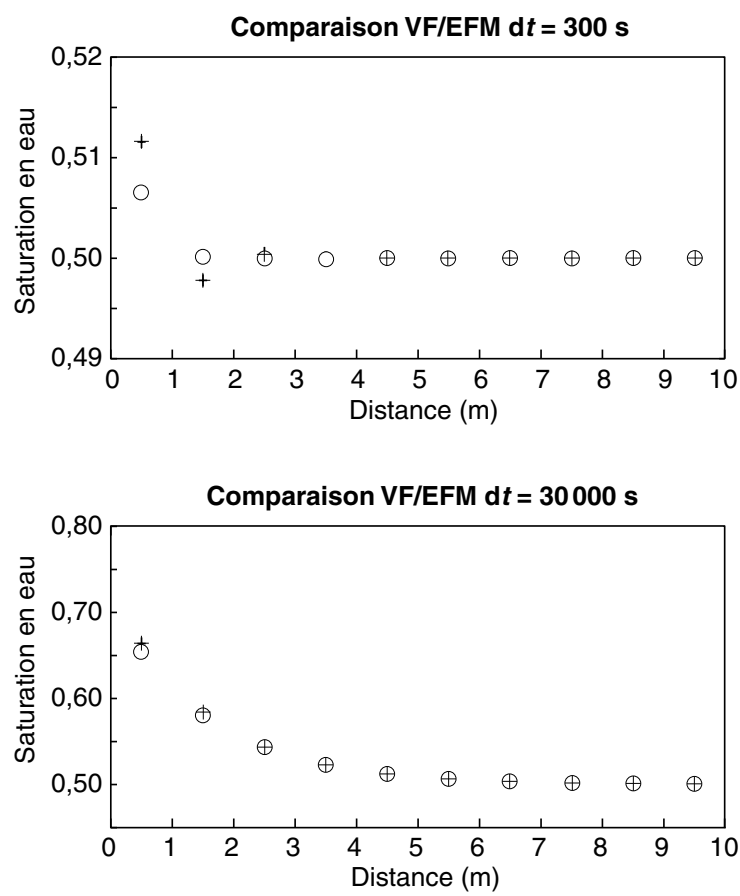

Figure 2

Comparaison des méthodes volumes finis (o) et éléments finis mixtes (+)

Comparison between finite volume method (o) and mixed finite element method $(+)$.

Les données physiques sont les masses volumiques de l'eau et de l'huile, qui s'expriment sous la forme: $\rho=$ $\rho_{0}\left(1+c\left(P-P_{0}\right)\right)$, et les données indiquées dans le tableau 2 . La perméabilité est supposée homogène et isotrope.

La perméabilité relative de chaque phase est fonction de la saturation de la phase :

$$
\left\{\begin{array}{l}
\text { si } S_{i}<S_{i o} \text { alors } k r_{i}=0 \\
\text { sinon } k r_{i}=k r_{i o}\left(\frac{S_{i}-S_{i o}}{1-S_{i o}}\right) a_{i o} \\
k r_{i o}, S_{i o} \text { et } a_{i o} \text { sont fixés }
\end{array}\right.
$$

Pour le cas test ci-dessus, $k r_{i o}=1, S_{i o}=0,2$ et $a_{i o}=1$ pour chacune des phases. La pression capillaire est supposée linéaire :

$$
P_{c}\left(S_{w}\right)=\frac{S-S_{i o}}{\alpha} \quad \text { avec } \quad \alpha=0,6
$$

TABLEAU 2

Données physiques Physical data

\begin{tabular}{c|c|c}
\hline Grandeur & Valeur & Description \\
\hline $\boldsymbol{K}$ & $1 \cdot 10^{-13} \mathrm{~m}^{2}$ & Perméabilité de la roche du faciès \\
\hline$\phi$ & $10 \%$ & Porosité de la roche \\
\hline$C_{o}$ & $1 \cdot 10^{-9} \mathrm{~Pa}^{-1}$ & Compressibilité du fluide $o$ \\
\hline$\mu_{o}$ & $1 \cdot 10^{-3} \mathrm{~Pa} \cdot \mathrm{s}$ & Viscosité du fluide $o$ \\
\hline$\rho_{0 o}$ & $1 \cdot 10^{+3} \mathrm{~kg} \cdot \mathrm{m}^{-3}$ & Masse volumique du fluide $o$ \\
\hline$P_{0 o}$ & $1 \cdot 10^{+6} \mathrm{~Pa}$ & Pression de référence $o$ \\
\hline$c_{w}$ & $1 \cdot 10^{-9} \mathrm{~Pa}-1$ & Compressibilité du fluide $w$ \\
\hline$\mu_{w}$ & $1 \cdot 10^{-3} \mathrm{~Pa} \cdot \mathrm{s}$ & Viscosité du fluide $w$ \\
\hline$\rho_{0 w}$ & $1 \cdot 10^{+3} \mathrm{~kg} \cdot \mathrm{m}^{-3}$ & Masse volumique du fluide $w$ \\
\hline$P_{0 w}$ & $1 \cdot 10^{+6} \mathrm{~Pa}$ & Pression de référence $w$ \\
\hline
\end{tabular}

Les conditions aux limites imposées sur le bord du maillage sont :

$$
\begin{array}{ll}
P_{w}(10, t)=15 \mathrm{bar} & S_{w}(10, t)=0,5 \\
\boldsymbol{u}_{w}(0, t)=0,05 \mathrm{~m} \cdot \text { jour }^{-1} & S_{w}(0, t)=0,8\left(P_{c}(0, \mathrm{t})=0\right)
\end{array}
$$

Les conditions initiales sont :

$$
S_{w}(x, 0)=0,5 \quad P_{w}(x, 0)=10 \text { bar }
$$

Les deux méthodes donnent un résultat similaire, sauf aux temps courts. Le profil de saturation obtenu avec la méthode utilisant les EFM présente des oscillations aux temps courts, i.e. lorsque le pas de temps $\Delta t$ est grand devant le temps caractéristique de diffusion dû à la compressibilité des fluides : $\tau_{p}=\frac{c \mu L^{2}}{K}$ et inférieur au temps caractéristique de diffusion de la saturation: $\tau_{s}=\frac{\mu L^{2}}{K P_{c}}$.

Nous avons vérifié que cette instabilité disparaît dès que $\Delta t$ est supérieur ou de l'ordre de $\tau_{s}$. La discrétisation VF ne présente pas cette instabilité, dont la présence est due à la perte du principe du maximum aux temps courts dans l'approche EFM (annexe).

\section{Ordre de grandeur}

On peut calculer les temps caractéristiques pour lesquels une instabilité de ce type peut apparaître. Le temps caractéristique de diffusion de la pression capillaire est : $\tau_{s}=\frac{\mu L^{2}}{K P_{c}}$. Pour une viscosité de l'ordre de $10^{-3} \mathrm{~Pa}$, une pression capillaire de $10^{5} \mathrm{~Pa}$, une perméabilité de $10^{-13} \mathrm{~m}^{2}$, et une longueur caractéristique de maille de l'ordre de $100 \mathrm{~m}$, on obtient un temps caractéristique supérieur à 100 jours. Nous avons vérifié que des instabilités sont susceptibles de se développer pour un tel pas de temps. 
Si l'on souhaite travailler aux temps courts, la gestion du pas de temps s'avère donc plus délicate avec la discrétisation EFM : des instabilités numériques peuvent apparaître pour des cas diphasiques compressibles avec pression capillaire. Néanmoins, une telle approche permet d'obtenir des solutions de référence sur des maillages très déformés.

\subsection{2 Étude d'un maillage fortement déformé}

On réalise un balayage par de l'eau d'un réservoir contenant de l'huile. Le maillage triangulaire est très déformé (fig. 3a). La triangulation utilisée possède une longueur caractéristique suivant l'axe $x 100$ fois plus importante que suivant $y$. L'approche EFM permet de traiter ce maillage. Les données physiques sont identiques au cas précédent.

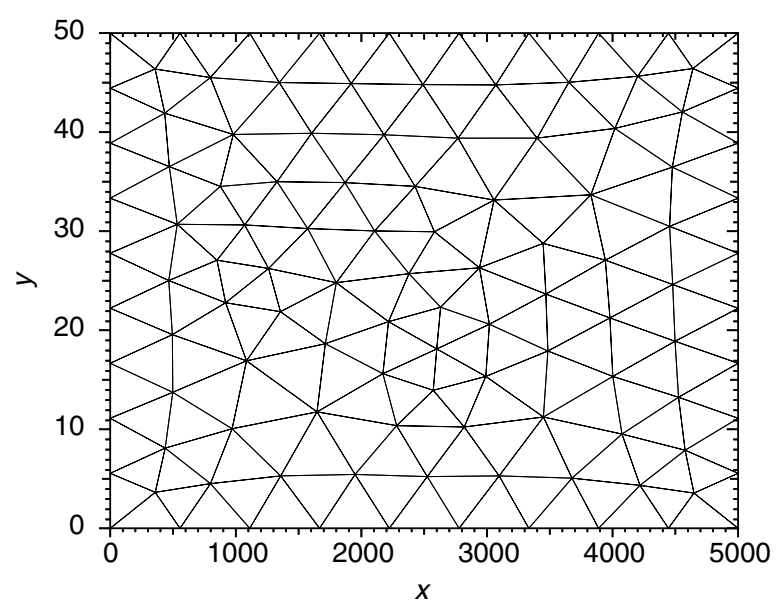

a Maillage



b Front de saturation

Figure 3

Avancée du front de saturation sur un maillage déformé.

Advance of the saturation front on a distorded grid.
Les conditions aux limites imposées sur le bord du maillage sont :

$$
\begin{array}{ll}
P_{w}(10, y, t)=15 \mathrm{bar} & S_{w}(10, y, t)=0,5 \\
\boldsymbol{u}_{w}(0, y, t)=0,95 \mathrm{~m} \cdot \text { jour }^{-1} & S_{w}(0, y, t)=0,8 \quad\left(P_{c}(0, t)=0\right)
\end{array}
$$

Les conditions initiales sont :

$$
S_{w}(x, y, 0)=0,5 \quad P_{w}(x, y, 0)=10 \text { bar }
$$

Comme dans le cas monophasique, l'approche EFM permet de traiter des maillages «critiques » très déformés. L'approche VF ne permet pas de traiter de tels maillages.

\section{CONCLUSIONS}

En écoulement monophasique, les tests comparatifs ont montré la convergence numérique des schémas volumes finis à continuité de flux en $\mathrm{O}$ à $2 \mathrm{D}$ et 3D (la convergence mathématique n'étant pas démontrée). Toutefois, si le maillage est trop déformé ou si les constrastes de perméabilité sont trop importants, des problèmes de convergence apparaissent.

Nous avons développé deux approches numériques pour modéliser les écoulements diphasiques de fluides compressibles sur maillages flexibles $2 \mathrm{D}$ et $3 \mathrm{D}$. L'utilisation des EFM pour discrétiser les termes paraboliques fournit des solutions de référence. Cette approche, plus robuste en ce qui concerne la discrétisation spatiale, permet de traiter des maillages très déformés et de valider la discrétisation volumes finis en $\mathrm{O}$, utilisée en pratique dans les simulations.

\section{RÉFÉRENCES}

Aarbogast, T., Wheeler, M.F. et Yotov, I. (1997) Mixed Finite Elements for Elliptic Problems with Tensor Coefficients as CellCentered Finite Differences. SIAM J. Numer. Anal., 34, 828-852.

Aavatsmark, I. (1996) Discretization on Non-Orthogonal, Quadrilateral Grids for Inhomogeneous, Anisotropic Media. J. Comput. Phys., 127, 2-14.

Aavatsmark, I. (1998a) Discretization on Unstructured Grids for Inhomogeneous, Anisotropic Media. Part I: Derivation of the Methods. SIAM J. Sci. Comput., 19, 1700-1716.

Aavatsmark, I. (1998b) Discretization on Unstructured Grids for Inhomogeneous, Anisotropic Media. Part II: Discussion and Numerical Results. SIAM J. Sci. Comput., 19, 1717-1736.

Aavatsmark, I., Barkve, T. et Mannseth, T. (1997) SPE Reservoir Simulation Symposium, 8-11 juin, Dallas, USA, SPE 38229.

Bergamashi, L., Mantanica, S. et Manzini, G. (1998) A Mixed Finite Element-Finite Volume Formulation of the Black-Oil Model. SIAM J. Sci. Comput., 20, 970-997.

Brezzi, B. et Fortin, M. (1991) Mixed and Hybrid Finite Element Methods, Springer Verlag.

Chavent, G. et Jaffré, J. (1986) Mathematical Models and Finite Elements for Reservoir Simulation, Studies in Mathematics and its Applications, North Holland.

Chavent, G., Jaffré, J., Eymard, R., Guerillot, D. et Weill, L. (1987) Discontinuous and Mixed Finite Elements for Two-Phase Incompressible Flow. SPE 16018.

Dong, Z., Eymard, R. et Hilhorst, D. (1999) Submitted. 
Durlofsky, L.J. (1993) A Triangle Based Mixed Finite ElementFinite Volume Technique for Modeling Two Phase Flow through Porous Media. J. Comput. Phys., 105, 252-266.

Eymard, R., Gallouët, T. et Herbin, R. (1996) Méthode des volumes finis, notes de cours DEA Analyse numérique.

Faille, I. (1992) Modélisation bidimensionnelle de la génèse et de la migration des hydrocarbures dans un bassin sédimentaire. Thèse, Université Joseph Fourier, Grenoble I.

Gallouët, T. (1999) Communication personnelle.
Lemonnier, P. (1991) Modélisation de l'écoulement appliquée à l'étude des réservoirs pétroliers. Cours DEA Méthodes quantitatives des Géosciences.

Pfertzel, A. (1987) Sur quelques schémas numériques pour la résolution des écoulements multiphasiques en milieu poreux. Thèse, Université de Paris VI.

Wheeler, M. (1997) in Transport de contaminants multiespèces en milieux poreux, 2-5 juin, INRIA, Rocquencourt.

Manuscrit définitif reçu en janvier 2000 


\section{ANNEXES}

\section{Définition d'une $M$ matrice}

Une matrice A est dite de type $M$ si et seulement si les coefficients $a_{i j}$ de A vérifient :

$$
\left\{\begin{array}{l}
a_{i i}>0 \text { pour tout } i \\
a_{i j} \leq 0 \text { pour tout } i \neq j \\
\left|a_{k k}\right| \geq \Sigma_{j \neq k}\left|a_{k j}\right| \text { pour tout } k \\
\text { il existe } k_{0} \text { tel que }\left|a_{k_{0} k_{0}}\right|>\Sigma_{j \neq k_{0}}\left|a_{k_{0} j}\right|
\end{array}\right.
$$

\section{Le schéma en $\mathrm{O}$ à $\mathbf{2 D}$ et $3 \mathrm{D}$}

À $2 \mathrm{D}$, on suppose que la pression varie linéairement par morceaux à l'intérieur de chaque maille, ou plus exactement qu'elle est linéaire sur chacune des quatre régions délimitées en pointillés (les mailles sont délimitées par des traits pleins) (fig. 1).

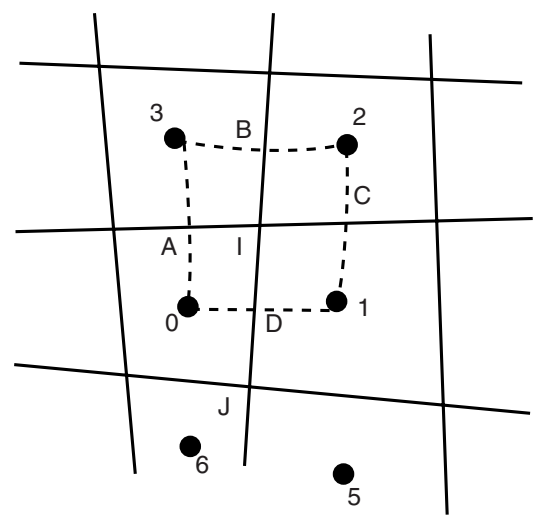

Figure 1

Maillage quadrangle 2D.

Quadrangular 2D grid.

La pression varie linéairement sur chacun des quadrilatères $0 \mathrm{ADI}, 1 \mathrm{DCI}, 2 \mathrm{CBI}, 3 \mathrm{ABI}$, les centres des mailles étant repérés par $0,1,2$ et 3 . Sur 0ADI (sur la figure 4 , D est le milieu de [IJ], I, J sommets de la maille de centre 0), la pression $\phi$ s'écrit à l'aide des fonctions linéaires $N_{0}, N_{A}$ et $N_{D}$ (ce sont, sur le triangle $0 \mathrm{AD}$, les fonctions de base linéaires pour les éléments finis triangulaires) :

$$
\phi=\phi_{0} N_{0}+\phi_{A} N_{A}+\phi_{D} N_{D}
$$

Le schéma en $\mathrm{O}$ construit une approximation consistante du flux à partir des hypothèses précédentes. Le flux entre les mailles de centre 0 et de centre 1 est la somme de deux termes que nous appellerons flux partiel relatif à ID et flux partiel relatif à $D J$. On calcule les flux en appliquant les continuités de la composante normale du flux dans le volume appelé région d'interaction (limité en pointillés sur la figure) sur chacune des demi-arêtes ID, IA, IC et IB. On obtient donc quatre équations, ce qui permet d'éliminer les inconnues secondaires
$\phi_{A}, \phi_{B}, \phi_{C}$ et $\phi_{D}$. Cette alternative constitue la méthode en $O$ (en fait, il n'est pas possible de satisfaire à la fois toutes les conditions de continuité de flux et de pression; le choix de fonctions de base linéaires rend le système surdéterminé).

Le flux sur ID comme sur chacune des demi-arêtes s'écrit, après élimination des inconnues secondaires, en fonction de la pression au centre des mailles.

Ce schéma se généralise à 3D pour des maillages de polyèdres de type quelconque. Pour le calcul de l'approximation du flux à travers une face du maillage, on évalue des flux partiels relativement à chaque sommet d'une face. Le calcul d'un flux partiel fait intervenir toutes les mailles contenant le sommet relatif au flux partiel.

\section{Principe du maximum et éléments finis mixtes}

Nous avons vérifié sur un cas simple qu'une méthode par éléments finis mixtes pour une équation de diffusion classique ne vérifiait pas le principe du maximum aux temps courts, contrairement à un schéma volumes finis.

Le principe du maximum assure la stabilité de la solution :

$$
\begin{gathered}
\mathcal{H} \in \mathcal{M}_{n}(\boldsymbol{R}), \boldsymbol{u} \in \boldsymbol{R}^{n} \\
(\mathcal{H} u \geq 0 \Rightarrow u \geq 0) \Leftrightarrow \mathcal{H}^{-1} \geq 0
\end{gathered}
$$

Considérons le maillage 1D de longueur 3. On étudie la réponse à un échelon de pression aux temps courts en discrétisant l'équation de diffusion classique en volumes finis et en éléments finis mixtes :

$$
\left\{\begin{array}{l}
\frac{\partial}{\partial t}(P)-\Delta P=0 \\
\frac{\partial}{\partial x} P(0,0)=\frac{\partial}{\partial \mathrm{x}} P(3,0)=0 \\
\left\{\begin{array}{lll}
P(x, t)=1 & \text { si } & x<1 \\
P(x, t)=0 & \text { si } & x \geq 1
\end{array}\right.
\end{array}\right.
$$

La discrétisation de l'équation de diffusion en éléments finis mixtes s'écrit :

$$
\left(\begin{array}{cc}
\mathcal{A} & \mathcal{B} \\
{ }^{t} \mathcal{B} & \mathcal{C}
\end{array}\right)\left(\begin{array}{l}
\mathcal{U} \\
\mathcal{P}
\end{array}\right)=\left(\begin{array}{l}
\mathcal{F} \\
\mathcal{G}
\end{array}\right)
$$

avec $\mathcal{C}=I / t$ ( $I$ est la matrice Identité). $\mathcal{A}$ est la matrice de raideur des vitesses.

On met ce problème sous la forme $\mathcal{H} \mathscr{P}=\mathcal{D}$ (le choix des conditions initiales assure $\mathcal{D}=\mathcal{G}$ et $\mathcal{G}$ positive).

Pour l'approximation éléments finis mixtes :

$$
\mathcal{A}_{E F M}=\left(\begin{array}{cccc}
1 & 0 & 0 & 0 \\
\frac{1}{6} & \frac{2}{3} & \frac{1}{6} & 0 \\
0 & \frac{1}{6} & \frac{2}{3} & \frac{1}{6} \\
0 & 0 & 0 & 1
\end{array}\right) \quad \mathcal{B}=\left(\begin{array}{ccc}
0 & 0 & 0 \\
-1 & 1 & 0 \\
0 & -1 & 1 \\
0 & 0 & 0
\end{array}\right)
$$


Pour l'approximation volumes finis :

$$
\mathcal{A}_{\mathcal{V F}}=\left(\begin{array}{llll}
1 & 0 & 0 & 0 \\
0 & 1 & 0 & 0 \\
0 & 0 & 1 & 0 \\
0 & 0 & 0 & 1
\end{array}\right) \quad \mathcal{B}=\left(\begin{array}{ccc}
0 & 0 & 0 \\
-1 & 1 & 0 \\
0 & -1 & 1 \\
0 & 0 & 0
\end{array}\right)
$$

On obtient l'équation (3) ci-dessous. En volumes finis, lorsque l'approximation du flux à deux points est consistante, la matrice est une $M$ matrice. Au temps courts, le principe du maximum est donc assuré. $\mathcal{H}_{\mathcal{V} \mathcal{F}}^{-1}$ est une matrice positive.

Quand $u \rightarrow \infty$ (pour les temps courts), on perd le principe du maximum avec la discrétisation EFM (les termes de $\mathcal{H}_{\mathcal{E F M M}^{-1}}$ ne sont pas tous positifs).

$$
\begin{aligned}
& \mathcal{H}_{\mathcal{E F M}}={ }^{t} \mathcal{B A}_{\mathcal{E}_{\mathcal{E F M}}^{-1} \mathcal{B}+u I=}\left(\begin{array}{ccc}
1,6+u & -2 & 0,4 \\
-2 & 4+u & -2 \\
0,4 & -2 & 1,6+u
\end{array}\right) \\
& \mathcal{H}_{\mathcal{V F}}={ }^{t} \mathcal{B A}_{\mathcal{V F}}^{-1} \mathcal{B}+u I=\left(\begin{array}{ccc}
1+u & -1 & 0 \\
-1 & 2 u & -1 \\
0 & -1 & 1+u
\end{array}\right) \\
& \mathcal{H}_{\mathcal{V F}}^{-l}=\frac{1}{\operatorname{det}\left(\mathcal{H}_{\mathcal{V F}}\right)}\left(\begin{array}{ccc}
(2+u)(1+u)-1 & (1+u) & 1 \\
(1+u) & (1+u)(1+u) & (1+u) \\
1 & (1+u) & (2+u)(1+u)-1
\end{array}\right) \\
& \mathcal{H}_{\mathcal{E F M}}^{-1}=\frac{1}{\operatorname{det}\left(\mathcal{H}_{\mathfrak{E F} \mathcal{M}}\right)}\left(\begin{array}{ccc}
(1,6+u)(4+u)-4 & 2(1,6+u)-0,8 & 4-0,4(4+u) \\
2(1,6+u)-0,8 & (1,6+u)(1,6+u)-0,16 & 2(1,6+u)-0,8 \\
4-0,4(4+u) & 2(1,6+u)-0,8 & (1,6+u)(4+u)-4
\end{array}\right)
\end{aligned}
$$

